up, they are sent to the front. Louvain as it was and Louvain as it is are scarcely more alike than are typhoid in times of peace and typhoid in time of war.

Typhoid is, of course, already taking part in the present war. Given the allied armies and the German army in the western theater, how should there not be typhoid? The only question is, How much more will there be a month hence? The lateness of the year, happily, will kill off flies, which are great carriers of the disease; but the flies are not yet gone, and they will more or less repeat that deadly part which they took in the South African war. There is plenty of the disease for them to carry. "It is well known," says Sir Almroth Wright, in the Times, September 28th, "that the infec tion of typhoid is thickly sown all along the frontier of France and Germany." Besides, among two millions of men, there are bound to be some who have the germs of the disease in them. And what is the good of talking of "sanitation," as if our men could have the warm baths and the water-closets of the average Englishman's home? Let us take $\mathrm{Dr}$. Johnson's advice, and clear our minds of cant. "An army," as Wright says, "on going out on active service goes from the sanitary conditions of civilization straight back to those of barbarism. . . . In war the doors are everywhere opened wide, both to the direct conveyance of infection by the excreta, and to its indirect conveyance by means of contaminated water. . . . On service, proper sanitary arrangements are very often impossible. We may think, for instance, of the situation of men in the trenches under fre. . . . When it comes to a tight place the alternative which will present itself will be that of drinking a polluted water or none. will, by the very nature of the case, be out of question to apply ordinary sanitary measures in an effective manner. - Infection spreads not only among the troops in the field, but also among the soldiers in hospital."

The Daily Chronicle, October 3rd, says: "It is re ported that 800 Germans, on the lines between Brussels and Antwerp, are suffering from typhoid fever."

That the protective treatment is indeed protective we all know. It is the experience of the nations of the earth; and the whole world is agreed about it. France, India, Canada, the United States, Italy, let alone ou enemies, are of one mind. Take only four instances : 1. British Army in India.- "The histories," as re gards typhoid fever, of 19,314 soldiers, whose average period of service abroad was twenty months, were care fully followed, and every precaution possible was take to verify the diagnosis bacteriologically. Of this number 10,378 were inoculated, and 8,936 not inoculated. The case incidence of typhoid fever among the inoculated was 5.39 per 1,000, and among the non-inoculate 30.4 per 1,000.-Report of Anti-Typhoid Committee, 1912.

2. United States Army.- "Inoculation was made compulsory in the American army in 1911, and has practically abolished the disease. In 1913 there were only three cases and no deaths in the entire army of ove 90,000 men."-Sir W. Leishman, British Medical ,Journal, August 22nd, 1914.

\section{A Direct Reading Psychrometer} By F. W. L. Peebles

EvenY weather bureau observatory uses a "wet and dry bulb" thermometer for the determination of the relative humidity of the atmosphere. The standard instrument is made up of two ordinary thermometers, as nearly alike as possible. One of them has the bulb covered with wicking which dips in water so as to keep covered with wicking which dips in water so as to keep
the bulb wet. Water on the wet bulb evaporates and cools the mercury thus recording a lower temperature. The drier the air, the greater will be the evaporation and consequent reduction of temperature on the we bulb side. The relative humidity of the air is computed from this difference. The capacity of the air for moisture varies with the temperature so that tables are ture varies with the temperat
necessary for the determination.

In the present instrument, which was designed by the writer, the use of tables has been eliminated and the

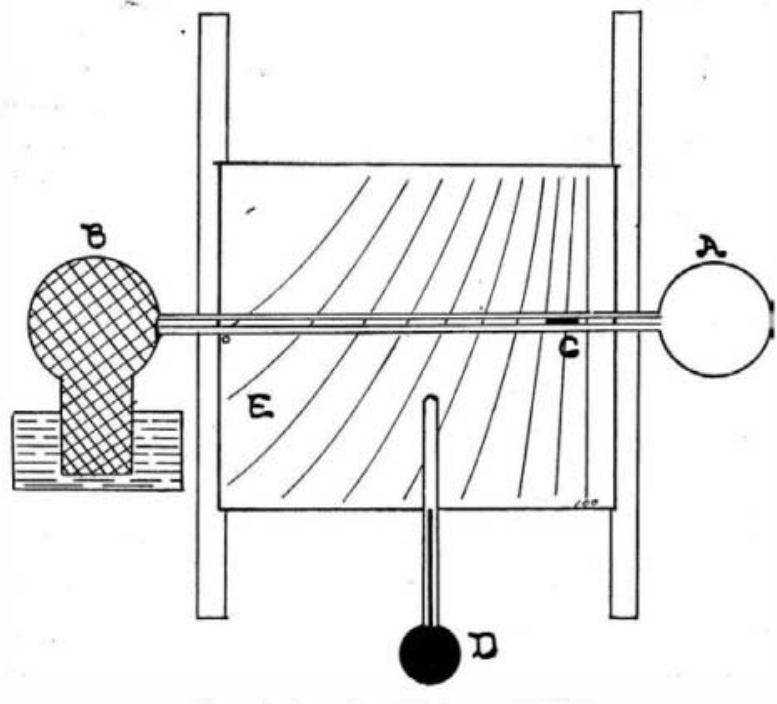

A Direct Reading Psychrometer.

readings are taken in per cent direct without any computation. Although it is believed that the device is original, it will not be patented, but rather, is hereby contributed to meteorological science by the designer.

In the figure $A$ is the dry bulb of an air thermometer, $\boldsymbol{B}$ is the wet bulb, $C$ is a plug of mercury, $D$ is an ordinary mercury thermometer for determining the air temperature and setting the sliding scale, and $E$ is a calibrated chart for taking the reading. The wick of the wet bulb is provided with a reservoir of water so that the bulb may be kept continuously wet. The sliding scale is set with reference to the thermometer $D$ so that a gauge point on it may be brought to the level of the top of the column thus setting the calibrated chart for the of the column thus setting the calibrated chart for the

If both bulbs are dry the indicating end of the plug of mercury will register with the line marked 100 per cent. This would be interpreted to mean that no evaporation is taking place; and, as far as the instrument is concerned, that the bulbs are both at the same temperature. Under this condition the position of the plug is ture. Under this condition the position of the plug is
the same for all temperatures of the air and consequently the 100 per cent line is vertical on the chart. When evaporation does take place the plug moves in a direction toward the cooler bulb, and this movement is a function both of the difference in temperature and of the absolut temperature of the outside air.

In order to eliminate the part that the temperature of the outside air plays, the sliding scale is adjusted to the height of the column of mercury in the auxiliary thermometer $D$. It may be well to note that such an arrangement is not a true differential thermometer, on account of the fact that the same difference in temperature will cause more motion of the plug when both are at low temperatures than when at high temperatures. This feature is particularly desirable on account of the greater capacity

In making up the instrument it is desirable that it be filled with a gas that is inert to mercury so that the plu is not acted upon in time. The bore of the connectin tube should be proportioned to the size of the bulbs so as to secure the right amount of motion to the plug. The tube should be of uniform cross section and the plug short so as to make the friction negligible.

In making up the instrument one bulb is sealed onto the tube and closed by the sealing, leaving only the opening to the tube. The second bulb is then sealed to the other end of the tube but this bulb is left with tail to be sealed off later. The whole system is then filled with the gas desired and a drop of mercury is added for the plug. The tail is now closed at some distance from the bulb and then this tail is sealed off near the bulb. The bulb that has the sealing end on it may now be used for the wet bulb end so that the wick will cover the point of closure.

To adjust the plug the dry bulb is held uppermost and in contact with the palm of the hand so that some of the gas is driven into the other bulb. The apparatus is then inverted so-that the mercury can enter the tube and the heated bulb allowed to cool so that the gas in the cooler bulb may enter the tube and drive the plug along before it. When the tiwo bulbs attain the same temperature the plug will come to rest. Should its position not be correct this may be changed by driving it back and using more or less heat as the case may be.

To calibrate the chart it is reccmmended that this be done by comparison with standard wet and dry thermometers and the table, although for those who have time for the mathematics it is easy to calculate the movement of the plug for different temperatures and differences in temperature of the two bulbs, and from this it is easy to calculate the relative humidity for any position of the plug. The rules for these computations may be found in any good text book in Physics.

\section{A Device for Regulating Storage Batteries from a Distance}

DURing the discharge of a storage battery the voltage gradually diminishes, so that, in order to maintain the constancy of lamps fed by the battery, it is necessary $o$ add a cell (or to cut one out, if the cells are connected in opposition) whenever the drop in voltage amounts to about two volts. This is usually done by moving a multiple commutator on the switchboard, an operation that involves considerable trouble when the switchboard is located in the cellar or in a distant part of the building. A reliable and easily constructed * From La Nature.
3. French Army.-In 1912 typhoid broke out in the barracks at Avignon. Of 2,053 men, 1,366 were protected and 687 were not. The non-protected had 155 cases, with 21 deaths; the protected had not one case. The protective treatment was made compulsory last winter in the French army; and, in special circumstances, among the reservists.-Lancet, January 4th, 1913.

4. Canadian-Pacific Railway.-Throughout the "railways camps" in Alberta, during 1911, among 5,500 protected there were only two cases of typhoid; among 4,500 non-protected there were 220 cases.-British Medi. cal Journal, June 6th, 1914

It remains to be noted: (1) That the vaccine contains no living germs of any sort. (2) That the treatment, though it gave good results in the South African war, has been improved since that time. (3) That the avoidance of exertion and excitement, on the day of treatment, is a great safeguard against any disturbance of the general health. (4) That, when time allows, it is always best to give the vaccine not all in one dose, but in two, or even three graduated doses, with a few days between each dose.

Sir Almroth Wright, by whose hands the gift of this treatment came to us, greatly desires that the treatment should be made compulsory, as in other armies, so in ours. Surely it is part of "my duty to my neighhor" that I should not, by having typhoid, expose him to the risk of infection from me. And it is certain that a soldier down with typhoid fever is not only useless against the enemy, but dangerous to his own friends.

device for operating the commutator from a distance by pressing a button is here shown. To the arm of the commutator is attached a disk, in the edge of which teeth of the shape illustrated have been cut with a fine saw or a file. The angle $d_{1} \bullet d_{2}$, subtended at the center of the disk by each tooth, is equal to the angle 504, through which the commutator turns in passing from one contact to the next.

The sucking coil $z$ is constructed as follows: The central tube is made of brass and is of square section, in order to prevent the square rod $D C$ that slides inside it from turning. The end pieces are of thick sheet brass, and have projections which are bent at right angles and perforated for the reception of screws by which the coil is fastened to the board. The winding contains about 1,200 feet of wire 1/60 inch in diameter. The sliding rod is composed of an iron segment $C$ and two brass segments, $D$ and $E$. The iron is attached rigidly to the brass, but the two brass segments are joined at $F$ by a flexible hinge, which allows the segment $E$ to be lifted easily.

When a current flows in the coil the iron rod is

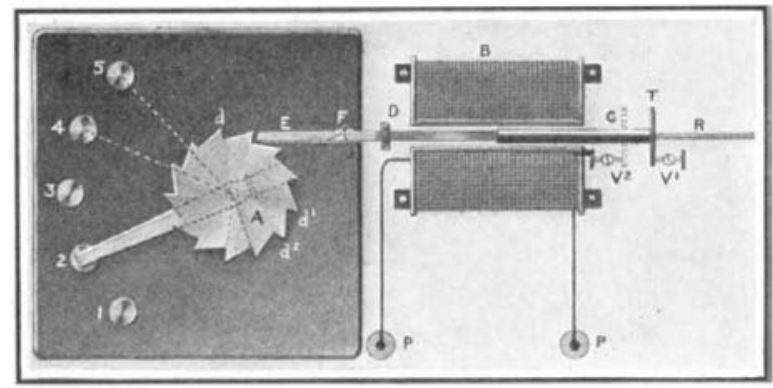

A device for regulating storage batteries from a distance.

drawn into the coil, carrying the brass rods before it In this movement the rod $E$ strikes a tooth of the disk and turns the disk through the angular interval between consecutive teeth, causing the commutator arm to advance from one contact to the next. When the current ceases the compound rod is drawn back by the spring $R$.

In this reverse motion the hinged segment $E$ rises above the next tooth and falls behind it, ready to push it forward when the current is again applied. The travel of the rod is limited by its cross-head $T$ striking the screw-tops $V_{1}$ and $V_{2}$, which are so adjusted that the commutator arm stops exactly in the middle of each commutator contact, and the end of the hinged segment $E$ falls behind the tooth with sufficient clearance.

The current may be taken from the storage battery itself by means of a branch circuit which includes as many cells as are required to energize the coil and turn the commutator. This circuit may be connected by very well insulated wires to push-buttons placed wherever they are wanted. When the lights grow dim another cell can be added to the battery by pressing a button. As the arm of the commutator is narrower than the space between consecutive contacts, the fact that the apparatus has operated is made evident, not only by the increase in brightness of the lamp, but atso by the slight tremor in the light caused by the momen tary interruption of the current. 